\title{
Ruta metodológica para el estudio de la formación integral desde los valores profesionales ${ }^{1}$
}

\author{
https://doi.org/10.21830/9789585287846.03
}

\begin{abstract}
Martha Hortensia Arana Ercilla², Vicente Hernán Ibarra Argoty 3 , Luis Carlos Pérez Ferro ${ }^{4}$, Erika Figueroa Pedreros', Jaime Alexander Fernández Camargo ${ }^{6}$, Diego Madrigal Pava ${ }^{7}$, Milton Fernando Monroy Franco ${ }^{8}$, Juvenal Diaz Mateus ${ }^{9}$
\end{abstract}

1 Este capítulo hace parte de los resultados del proyecto de investigación "La formación en valores del Profesional en Ciencias Militares del Ejército Nacional de Colombia. Un estudio del principio ético "disciplina militar" del Grupo de Investigación en Ciencias Militares de la Escuela Militar de Cadetes "General José María Córdova”, registrado con el código COL0082556 de Minciencias. Los puntos de vista y los resultados de este capítulo pertenecen a los autores y no reflejan necesariamente los de la institución participante.

2 Economista (Universidad de la Habana). PhD en Educación (Universidad Tecnológica de La Habana). Posdoctorado en Educación (Universidad Pedagógica de Colombia). Docente investigador de la Facultad de Ciencias Militares de la de Escuela Militar de Cadetes “General José María Córdova”. ORCID: https://orcid.org/0000-00024851-5279. Contacto: martha.arana@esmic.edu.co

3 Oficial de la Reserva Activa del Ejército Nacional de Colombia. Profesional en Ciencias Militares (Escuela Militar de Cadetes). Abogado (Universidad La Gran Colombia). Magíster en Análisis y Prevención del Terrorismo (Universidad Rey Juan Carlos, España). Docente investigador de la Facultad de Ciencias Militares de la Escuela Militar de Escuela Militar de Cadetes "General José María Córdova”. ORCID: https://orcid.org/0000-0003-43730225. Contacto: vicente.ibarra@esmic.edu.co

4 Psicólogo (Universidad Santo Tomás). Magíster en Educación (Pontificia Universidad Javeriana). Docente investigador en la Facultad de Ciencias Militares de la Escuela Militar de Cadetes "General José María Córdova”. ORCID: https://orcid.org/0000-0002-4793-7082. Contacto: luis.peres@esmic.edu.co

5 Historiadora (Universidad Industrial de Santander). Magíster en Historia Militar (Escuela Militar de Cadetes). Docente investigador en la Facultad de Ciencias Militares de la Escuela Militar de Cadetes "General José María Córdova”. ORCID: https://orcid.org/0000-0001-6241-344X. Contacto: erika.figueroa@esmic.edu.co

6 Ingeniero Informático (Escuela Militar de Aviación "Marco Fidel Suárez"). Oficial del Cuerpo de Vuelo de la Fuerza Aérea Colombiana. Jefe de Áreas de Formación en la Facultad de Ciencias Militares de la Escuela Militar de Cadetes "General José María Córdova”. ORCID: https://orcid.org/0000-0001-7732-6592. Contacto: jaime. fernandez@fac.mil.co

7 Profesional en Ciencias Militares (Escuela Militar de Cadetes). Administrador de Empresas (Universidad Militar Nueva Granada). Jefe de Formación Militar Académica en la Facultad de Ciencias Militares de la Escuela Militar de Cadetes "General José María Córdova”. ORCID: https://orcid.org/0000-0003-2997-3810. Contacto: diego.madrigal@esmic.edu.co

8 Profesional en Ciencias Militares (Escuela Militar de Cadetes). Magíster en Gestión de Proyectos (Universidad Militar Nueva Granada). Piloto Estandarizador de la Aviación del Ejército. Decano de la Facultad de Ciencias Militares de la Escuela Militar de Cadetes “General José María Córdova”. ORCID: https://orcid.org/00000002-9382-7239. Contacto: milton.monroy@esmic.edu.co

9 Profesional en Ciencias Militares (Escuela Militar de Cadetes). Magíster en Seguridad y Defensa Nacionales (Escuela Superior de Guerra, Colombia). Magíster en Estudios de Defensa (King's College London, Reino Unido). Magíster en Ciencias y Artes Militares, Estrategia (Escuela de Comando y Estado Mayor del Ejército de los Estados Unidos - Fort Leavenworth, Estados Unidos). Contacto: juvenal.diaz@buzonejercito.mil.co 
Las consideraciones que se presentan enseguida se refieren a las acciones, en el ámbito metodológico, de una experiencia investigativa sobre la formación integral del profesional oficial del Ejército de Colombia. Esto, visto desde el estudio de los principios y valores profesionales que le dan sentido al proceso educativo.

En primer lugar, y con el objeto de presentar la información y pertinencia del apartado, se indica, como ya se mencionó, que el marco temático de la investigación que le da soporte a la ruta, vierte su análisis en los procesos educacionales de las Fuerzas Militares, específicamente en el Ejército de Colombia y su Escuela Militar de Cadetes. Ello implica, que la reflexión sobre los procesos educativos de sus propios miembros, aún en formación de pregrado, ocupa un lugar privilegiado en la agenda de temas que potencian el desarrollo de la institución castrense; además, se convierte en insumo importante de ejercicios para la actualización curricular y, si fuere el caso, de revisión a los métodos y técnicas que se utilizan al momento de educar desde una mirada centrada en valores.

Los procesos de orden metodológico que se muestran son elementos adicionales a los hallazgos que en materia educativa ha realizado la Escuela Militar de Cadetes "General José María Córdova” durante la última década, los cuales han servido como conocimiento novedoso en la actualización de las propuestas curriculares que hoy están vigentes. Además, la suma del conocimiento hallado al preexistente provee a la institución de nuevo material académico que puede poner a disposición de los diferentes miembros de la comunidad educativa.

En síntesis, se proporcionan elementos investigativos de orden técnico, pero fundamentalmente empírico, al estudio de prácticas valorativas (análisis de valores) en el ámbito de la formación, mediante la propuesta de una ruta metodológica cuya descripción es el objeto de este apartado.

Lo que sigue incluye, específicamente, el proceso utilizado en la Investigación titulada: "La formación en valores del Profesional en Ciencias Militares del Ejército Nacional de Colombia. Un estudio del principio ético 'Disciplina Militar', para lo cual se definen aspectos del tipo de investigación, los procedimientos, las categorías, los instrumentos y técnicas, así como los participantes o sujetos de estudio. 


\section{Aspectos generales de la ruta metodológica}

La intención genuina de la ruta propuesta, es proporcionar al ámbito académico herramientas predominantemente procedimentales para el estudio de los valores profesionales dentro del contexto educativo. Dada la especificidad del ambiente militar, la ruta podría ser una interesante forma de ampliar el abordaje de los currículos castrenses y, particularmente, el del profesional en Ciencias Militares.

Sin embargo, lo que se describe relata el paso a paso de la experiencia investigativa, enunciada de manera sucinta anteriormente, y busca mostrar la el modo mediante el cual, en un escenario con un componente de doctrina robusto, se manifiestan los valores de formación profesional. Así, la ruta siempre tendrá como referente primario la investigación denominada: "La formación en valores del Profesional en Ciencias Militares del Ejército Nacional de Colombia. Un estudio del principio ético Disciplina Militar".

Se busca que la experiencia particular pueda ser empleada por otros investigadores en ejercicios similares de evaluación de los valores en su proceso de construcción, para que reconozcan la ruta y la puedan implementar en escenarios de educación superior, en general, o en instituciones militares, de manera particular.

Para el caso de estudio, los referentes pedagógicos y curriculares se fundamentan en el documento: "Proyecto Educativo del Programa Profesional en Ciencias Militares".

\section{Primer aspecto de la ruta: modelo, enfoque y alcance del ejercicio investigativo}

La investigación que dio lugar a esta reflexión metodológica incluyó dentro de sus objetivos la enunciación o formulación de una ruta para el abordaje de los valores profesionales y los valores específicos de estudio: valor, respeto y honestidad, que se tomaron a partir de dos documentos orientadores de formación, la doctrina militar y la especificidad de su concreción en el Proyecto Educativo del Programa de formación del Profesional en Ciencias Militares —en el que se precisan las competencias del currículo formal, de 
donde se recogen dichos valores en las dimensiones del ser y convivir-. Sumado a lo anterior, y de acuerdo con el desarrollo del proceso, se establecieron relaciones entre los valores seleccionados de los documentos referidos y las competencias generales de formación. De estas últimas se eligieron los valores "honestidad" y "respeto", que hacen parte integral del principio ético doctrinal "Disciplina Militar". El contenido de los dos valores fue determinado por las competencias específicas que constituyen sistemas de valores (ver figuras 1, 2 y 3). Así mismo, se relacionaron las competencias específicas con la enunciación de indicadores susceptibles de ser apreciados mediante los instrumentos de observación encubierta y técnica epistolar elegidos para tal fin (ver tablas 1, 2, 3 y 4), que hacen posible el triple diagnóstico, modo escogido para evaluar la formación de los valores. Luego, se hizo el tratamiento de la información por instrumento, se establecieron las relaciones entre ellos y se propuso, finalmente, la discusión educativa que cierra.

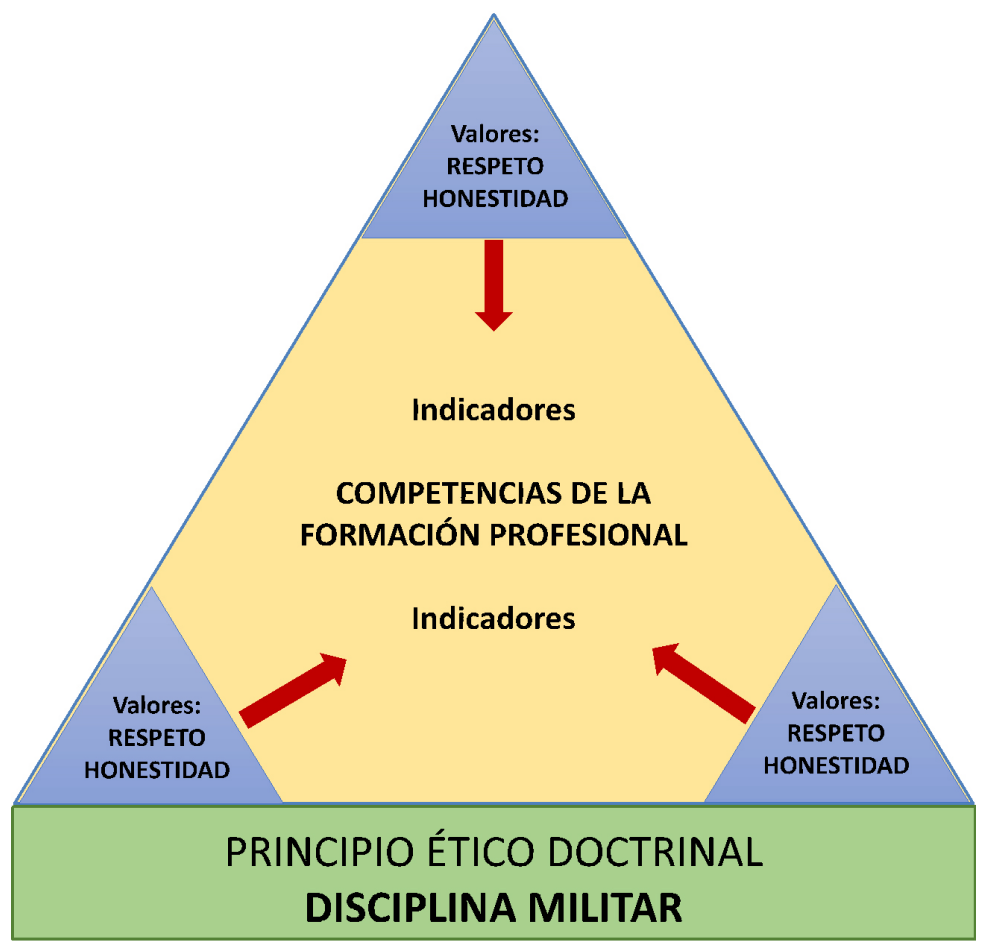

Figura 1. Modelo para la selección de los valores profesionales en la formación integral 
La figura muestra la interdependencia entre los principios y valores propios de la institución castrense y las competencias generales y específicas, que son parte de la formación profesional, que dan lugar a los indicadores de evaluación de los valores construidos por el equipo de trabajo. Lo descrito anteriormente es el resultado de pensar el camino para responder la pregunta de investigación: ¿cómo se manifiestan los valores profesionales de respeto y honestidad del principio ético disciplina militar, en el comportamiento de estudiantes de la Escuela Militar de Cadetes "General José María Córdova"?

El paradigma de investigación utilizado es cualitativo-interpretativo, con un enfoque hermenéutico-cultural. Se trata de una investigación que combina el triple diagnóstico participativo, el análisis documental, la observación participante encubierta y la utilización de técnicas de documentos personales, particularmente basadas en material epistolar (carta preescrita), y su alcance es de tipo descriptivo.

\section{Segundo aspecto de la ruta: consideraciones de procedimiento para la identificación de los valores de formación (respeto y honestidad)}

Lo siguiente se refiere a los momentos o pasos a seguir que el colectivo de investigadores ejecuta para identificar los valores a investigar, así como para medir o ponderar las categorías de análisis, hacer la sistematización de lo encontrado y la formulación de las implicaciones de los hallazgos, desde un análisis pedagógico y de la formación profesional militar. De acuerdo con lo anterior, se identificaron ocho momentos, cada uno con acciones específicas que enseguida se indican.

Tabla 1. Momentos del procedimiento

\begin{tabular}{ll}
\hline Momento 1 & $\begin{array}{l}\text { Determinación y fundamentación de los conceptos objetos de investiga- } \\
\text { ción: valores profesionales, formación integral y currículo. }\end{array}$ \\
Momento 2 & $\begin{array}{l}\text { Exploración y elección de los valores profesionales desde el principio } \\
\text { disciplina militar: valores honestidad y respeto. }\end{array}$ \\
Momento 3 & $\begin{array}{l}\text { Definición del contenido de los valores seleccionados desde el proyecto } \\
\text { educativo del programa de formación profesional. }\end{array}$ \\
\hline
\end{tabular}


Momento 4 Relación del valor elegido con los valores de las competencias generales y específicas de formación, para la construcción del sistema de valores que expresa sus contenidos.

Momento 5 Establecimiento del contenido de cada valor a partir de las competencias específicas de este, así como de los indicadores cualitativos o cuantitativos que permiten visibilizar, valorar y evaluar los valores desde la actividad formativa estudiada.

Momento 6 Estimación de los niveles de desarrollo de los valores profesionales que se manifiestan en las actitudes y comportamientos observados en los estudiantes.

Momento 7 Comparación de la descripción de la manifestación de los valores estudiados con el currículo institucional establecido.

Momento 8 Caracterización pedagógica de la educación en valores profesionales y la formación integral objeto de estudio.

A continuación, se desglosan los momentos antes señalados para ampliar y clarificar la ruta metodológica del estudio de los valores.

Momento 1. Determinación y fundamentación de los conceptos objetos de investigación: valores profesionales, formación integral y curriculo

En este momento se pone de relieve la necesidad de una fundamentación teórica y conceptual de las categorías que se establecen y se desarrollan posteriormente. Lo que ratifica que toda investigación, aún de corte descriptivo, requiere de un posicionamiento científico para su realización. Para esta investigación, los conceptos tratados son: valores profesionales, formación integral y currículo.

Momento 2. Exploración y elección de los valores profesionales desde el principio disciplina militar: valores honestidad y respeto

El Ejército Nacional de Colombia define los principios y valores como aquellas conductas que, además de regir, dejan una impronta de moralidad y ética a la actuación de sus integrantes. Para efectos de la presente ruta, es necesario limitar su alcance desde los valores que pueden ser observables en los estudiantes en formación de la Escuela Militar de Cadetes.

Para la determinación de los valores de estudio se asumió la consideración de que estos constituyen un sistema, es decir que cada valor se halla en inte- 
racción con otros valores que le dan su propio contenido. Para algunos epistemólogos que desarrollan el sistemismo, todo concepto es un sistema, pues se expresa a través del todo y sus partes, a la vez que cada parte puede ser un todo y constituirse de otras partes. Un ejemplo de ello es el valor "responsabilidad" - cuyo contenido está en interacción sistémica respecto a los valores: "sinceridad", "diálogo", "constancia”, "optimismo", "confianza” y "respeto", entre otros_- en dependencia de la comprensión de este y del ámbito y alcance de su desarrollo educativo. Para Sandoval (2007), los valores de un individuo o de una colectividad no se presentan aislados, yuxtapuestos o desordenados, por el contrario, están interrelacionados y son interdependientes.

La existencia de un sistema de valores implica que la educación en valores sea igualmente un proceso sistémico, pluridimensional e intencional, que garantice la formación y el desarrollo de la personalidad consciente (Arana y Batista, 1999), constituyéndose así en el principal medio mediante el cual las dimensiones del ser y el convivir se fortalecen, a través de un modelo pedagógico basado en competencias que busca cultivar en los alumnos las aptitudes, comportamientos y conocimientos que se estiman necesarios para su adecuado desempeño profesional (ESMIC-PEP, 2010).

Tal desempeño no sería posible sin un modelo educativo a través del cual

el ser humano sepa que, si es verdad que tiene un proyecto personal de vida que quiere desarrollar profundamente, [...] necesita, así mismo, desarrollar una conciencia moral autónoma partiendo de un sistema de valores seleccionados, elegidos y acogidos como norma de conducta. (Tierno, 1996, p. 198)

Este sistema de valores, concebido como un modelo de formación de competencias particulares, incluye, además de los valores de alta significación y universalmente aceptados, aquellos que son de carácter específico a la profesión, y que se convierten en la base de una sociedad, profesión u organización determinada.

La existencia de una relación explícita o implícita en la educación del valor puede llevarse a cabo a través del más mínimo acto educativo, sin alguna referencia a un conjunto de valores, pues ellos siempre están presentes en las relaciones humanas (Guervilla, 1994). Al estar interrelacionados entre sí, los valores son holísticamente fortalecidos al interior del 
claustro educativo; sin embargo, allí no podrán observarse en su totalidad, pues la exteriorización de algunos requerirá de unas circunstancias específicas, en la que el individuo, al enfrentar auténticos riesgos, demostrará el aprendizaje y su capacidad para la aplicación de los mismos.

Los valores de la formación del profesional en Ciencias Militares se toman del Manual Fundamental del Ejército MFE 1-0 (2016), que limita a diecisiete los principios y valores institucionales, los cuales define como "reglas de conducta que se encuentran en la base de la actuación del Ejército y que animan, dan sentido moral y ética institucional a sus actos" (p. 25). Apelar a la totalidad de los principios y valores institucionales para el desarrollo de la investigación se hace imposible, pues amplía demasiado el objeto de estudio, lo que impide abarcarlos todos; no obstante, cabe anotar aquí cuáles son, se trata de: respeto por los DD. HH. y acatamiento del DIH, respeto por la constitución y la ley, honor militar, disciplina, ética en todas las actuaciones, compromiso, fe en la causa, persistencia en el empeño, respeto, honestidad, lealtad, valor, prudencia, constancia, solidaridad, fidelidad y transparencia. Para la selección de los valores objeto de estudio se tomó en cuenta su relación con la convivencia y la posibilidad de ser observables en el nivel de la formación.

Inicialmente, el equipo se enfrentó a los diecisiete principios y valores, sin embargo, por la imposibilidad de abarcarlos, como se explicó anteriormente, se decidió elegir un principio ético doctrinal fundamental que, una vez aplicado sistemáticamente en el individuo a través del entrenamiento y la educación militar, sea eventualmente adoptado de manera consciente como un valor, contribuyendo así a la adaptación social de las personas a un entorno específico, por medio de una influencia directa en la autorregulación de la conducta (Sánchez, 2005).

La selección del principio "disciplina militar" se realizó bajo la perspectiva de criterios metodológicos, de contenido formativo y de experticia práctica. Bajo este principio se identificaron los valores de "respeto" y "honestidad". Siendo la "disciplina" el principio a observar y analizar en la formación integral, teniendo en cuenta los siguientes argumentos:

- La disciplina, como elemento fundamental en toda organización militar, no solo es un principio para el Ejército Nacional, sino 
también, un valor adoptado por cada uno de los individuos. Esto quiere decir que su expresión se da no solamente desde la organización militar hacia el individuo, sino también al interior del mismo individuo, que modifica conscientemente su comportamiento para ajustarlo a unas nuevas condiciones de vida.

- La disciplina, al agrupar en sí otros principios y valores, se erige jerárquicamente por encima de los demás y, por tanto, se establece como principal elemento a observarse en la formación estudiada.

- La predominancia en el uso del principio disciplina, puesto que está presente de manera permanente en todos los actos del servicio como fuera de él, es un elemento característico y fundamental de la profesión militar.

- La disciplina se presenta, a través de la doctrina, como el principal elemento organizador y aglutinador para el liderazgo eficaz y el mando tipo misión. La iniciativa disciplinada es adoptada por el Ejército Nacional como una nueva filosofía de mando, que es aplicada mediante una relación de confianza en la cual los líderes dictan las directrices generales de la misión, para que subalternos establezcan el planeamiento detallado en relación con el tiempo y los recursos disponibles.

La elección del principio ético "disciplina militar" como principio clave y objeto de estudio de la formación integral profesional, no solo pretende limitar las diferentes comprensiones que sobre la disciplina existen, pues el enfoque desde lo militar la delimita y la asocia a las particularidades del profesional que se forma, sino que también implica investigar de manera directa el rasgo fundamental de la formación militar integral de los oficiales del Ejército Nacional.

\section{Momento 3. Definición del contenido de los valores seleccionados desde el proyecto educativo del programa de formación profesional}

Como se destaca en el momento anterior, el estudio se fundamenta en los principios y valores institucionales de la formación del profesional en Ciencias Militares, regidos por la doctrina militar vigente y recogidos como exigencias curriculares en el proyecto educativo del programa de formación. La propuesta parte del currículo allí expresado, que sirvió como fuente para la selección de 
los valores relacionados con la disciplina militar. A continuación, se muestra el listado de valores del currículo y sus definiciones, así como los criterios seguidos para la elección de los valores "respeto" y "honestidad" (ESMIC-PEP, 2019).

1. Respeto: profunda consideración por todas las personas y su dignidad, los compañeros superiores, por sí mismo y su familia.

2. Honestidad: actuar con decencia, decoro, compostura, honradez e integridad de acuerdo con su conciencia.

3. Lealtad: sentimiento de respeto a los principios morales propios, a los compromisos establecidos o hacia alguien. Se relaciona con las personas, los superiores, compañeros y subalternos.

4. Valor: coraje y osadía para enfrentar los desafíos y retos que la misión impone, así como para reconocer los errores y decidirse a rectificar.

5. Prudencia: sabiduría práctica para ejecutar y tomar decisiones acertadas en diferentes situaciones.

6. Constancia: actitud y hábito permanente, sin interrupción, persistencia, tenacidad y perseverancia para obtener los objetivos.

7. Solidaridad: responder con acciones humanitarias ante situaciones que pongan en peligro la vida, la paz, el orden y la seguridad de la población.

8. Fidelidad: firmeza y constancia en los afectos, ideas y obligaciones, así como en el cumplimiento de los compromisos establecidos. Se relaciona con las instituciones, la patria, la familia, la Constitución y la ley.

9. Transparencia: honor y rectitud en las acciones que se emprenden por la patria, siendo impenetrables ante la corrupción y actuando según la Constitución Nacional y la ley, bajo el lema: "La ética y la integridad me hacen transparente".

Dada la cantidad de valores, se consideró que no era posible estudiarlos todos de manera independiente; además, algunos de ellos no se manifestaban en el ámbito de estudio, pero por sus interrelaciones con el principio "disciplina militar" era posible agruparlos, de manera tal que los escogidos para la investigación contuvieran elementos fundamentales de otros. Como resultado del análisis del grupo de investigación se eligieron tres, los que representaban 
el principio ético "disciplina militar" y cumplieran los criterios de selección antes planteados, estos fueron: respeto, honestidad y transparencia. Después de varias deliberaciones se decidió incluir la transparencia en la honestidad, para, finalmente, elegir solamente dos valores: respeto y honestidad.

Momento 4. Relación del valor elegido con los valores de las competencias generales y especificas de formación, para la construcción del sistema de valores que expresa sus contenidos

Luego de seleccionar los valores de estudio relacionados con la disciplina, según el criterio de los investigadores, se establece su contenido desde la relación con las competencias generales del currículo de formación, lo que conforma un sistema de valores de respeto y honestidad. Así se establece la relación del valor elegido para el estudio con los valores que se señalan en las competencias generales de formación del profesional, que se constituyen en un sistema para expresar el contenido del valor en estudio. A continuación, se muestran las figuras que expresan el sistema de cada valor.

Así, el valor profesional seleccionado expresa su contenido a través de los valores de las competencias generales. A su vez, estos valores del sistema expresan su significado desde las competencias específicas, que permiten operacionalizar los valores en indicadores para el estudio.

\section{SISTEMA DEL VALOR "RESPETO" DEFNIDO DESDE LAS COMPETENCIAS GENERALES}

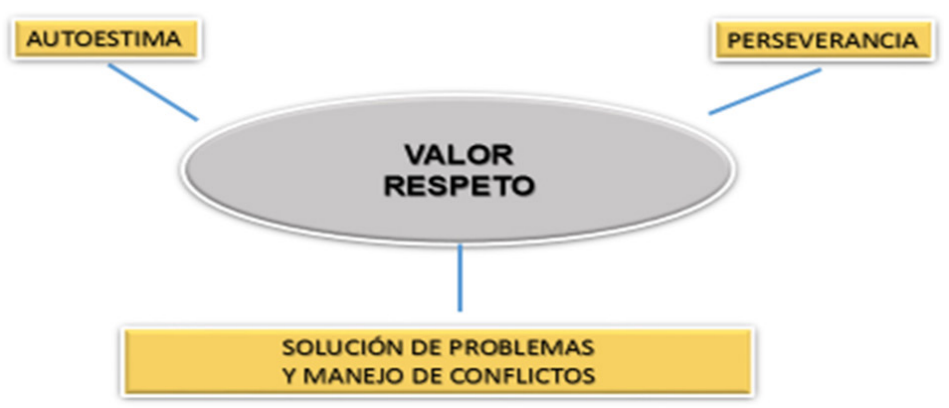

Figura 2. El valor "respeto" definido desde las competencias generales descritas en el currículo de formación y su sistema de valores 


\section{SISTEMA DEL VALOR “HONESTIDAD” DESDE LAS COMPETENCIAS GENERALES}

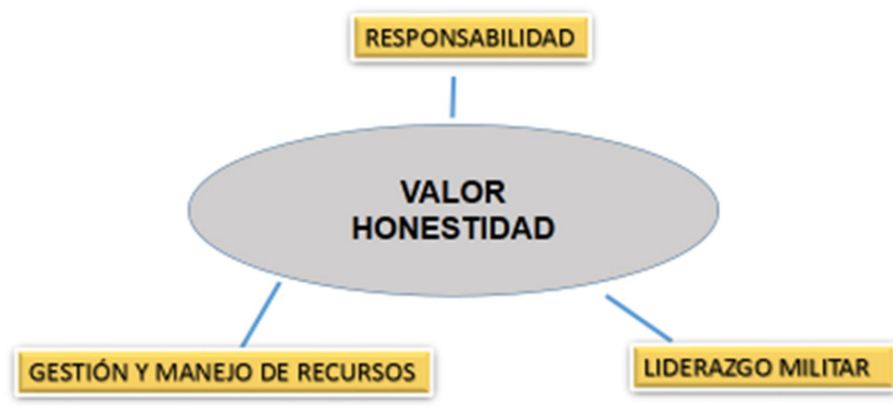

Figura 3. El valor "honestidad" definido desde las competencias generales descritas en el currículo de formación y su sistema de valores

Momento 5. Establecimiento del contenido de cada valor a partir de sus competencias especificas y de sus indicadores cualitativos o cuantitativos, que permiten visibilizar, valorar y evaluar los valores desde la actividad formativa estudiada

Como se ha dicho anteriormente, el valor profesional seleccionado expresa su contenido a través de los valores de las competencias generales. A su vez, estos valores expresan su significado en competencias específicas, las cuales permiten operacionalizar los valores en indicadores para el estudio de su comportamiento dentro del proceso de formación. A continuación, se muestran los indicadores extraídos de las competencias específicas del currículo de formación:

Tabla 2. Indicadores de evaluación de la formación del valor "respeto"

\section{Respeto}

Sistema de valores: perseverancia, autoestima y solución de problemas y manejo de conflictos.

\section{Indicadores}

1. Denota atención por el cumplimiento de las funciones propias del servicio.

2. Responde a los obstáculos, llamados de atención o problemas que se presenten en el servicio. 


\section{Respeto}

Sistema de valores: perseverancia, autoestima y solución de problemas y manejo de conflictos.

\section{Indicadores}

3. Corrige las novedades presentadas dando solución desde el conocimiento de la profesión.

4. Instruye con liderazgo el cumplimiento de las tareas a sus subalternos, compañeros y superiores.

5. Aplica los conocimientos adquiridos durante su formación profesional para el cumplimiento del servicio.

6. Evidencia en su comportamiento profesional el porte militar.

7. Propone soluciones creativas a las situaciones presentadas durante el servicio.

8. Muestra atención ante conflictos del servicio de forma respetuosa sin dejar de cumplir parámetros de seguridad.

9. Denota en el liderazgo del servicio buenas relaciones interpersonales basadas en la disciplina, el respeto y el mando.

Tabla 3. Indicadores de evaluación de la formación del valor "honestidad"

\section{Honestidad}

Sistema de valores: responsabilidad, gestión y manejo de recursos y liderazgo militar

Indicadores

1. Reconoce los señalamientos de los superiores, modificando la manera de actuar en sus funciones.

2. Cumple las órdenes impartidas en el servicio y las funciones dispuestas por el mismo.

3. Distribuye prioritariamente los recursos de acuerdo con las necesidades del servicio.

4. Controla el adecuado uso de los recursos.

5. Vela por el uso adecuado de los recursos humanos y materiales según las exigencias del servicio.

6. Muestra ejemplo al personal subalterno durante la ejecución de su servicio.

7. Expresa con claridad y firmeza las órdenes a cumplir por sus subalternos para prevenir posibles riesgos.

8. Incentiva a sus subalternos al cumplimiento de actividades del servicio, por medio del ejemplo y el buen trato. 
Momento 6. Estimación de los niveles de desarrollo de los valores profesionales que se manifiestan en las actitudes y comportamientos observados en los estudiantes

En este momento o paso se realiza una caracterización de la formación de los valores, desde una valoración, como resultado de la aplicación integrada de ambas técnicas (observación encubierta y técnica epistolar) que, en conjunto, permiten interpretar el constructo valor, como aquel conocimiento consciente en la actuación profesión del servicio realizado. Pues se analiza la interconexión de lo que piensa, dice y hace el estudiante, con lo cual se muestra el nivel de desarrollo y de educación del valor, según el comportamiento correspondiente con lo que se piensa y se dice de este.

Momento 7. Comparación de la descripción de la manifestación de los valores estudiados con el currículo institucional establecido

Este es un momento metodológicamente necesario para obtener resultados en el estudio, puesto que permite establecer, desde una mirada global y no solo la individual sobre cada estudiante, la tendencia de lo hallado acerca del alcance de la formación de los valores analizados. Consiste en comparar la descripción de la manifestación de los valores estudiados, desde los indicadores de análisis, con lo establecido en el currículo formal propuesto, lo que permite precisar las limitaciones pedagógicas y curriculares existentes y los aspectos por mejorar.

Momento 8. Caracterización pedagógica de la educación en valores

profesionales y la formación integral objeto de estudio

Por último, se realiza una valoración crítica de los resultados desde la pedagogía y el modelo pedagógico de la formación del profesional, delimitando recomendaciones necesarias para la mejora curricular, de la educación en valores y sus didácticas específicas, con el fin de consolidar una observación rigurosa con respecto a la formación integral.

\section{Tercer aspecto de la ruta: elección de los sujetos de investigación y uso de los instrumentos para el levantamiento de la información}

El ejercicio investigativo tuvo como marco metodológico el triple diagnóstico participativo. Este se fundamenta en la concepción educativa que ubica 
al sujeto de educación en el centro, de ahí que entienda la integralidad de los procesos y de la formación como factor decisivo. "La formación integral de los sujetos supone colocar a las personas en el centro del proceso, [...] a la relación entre la vida cotidiana y al proyecto al cual se aspira, conjugando lo individual y lo social" (Astorga, 1994).

De la Rúa (2013) reconoce que:

En el diagnóstico es necesario conocer no solo las concepciones que mueven a los sujetos participantes, sino cómo se mueven en la práctica específica que se estudia y cómo influyen las situaciones en las concepciones y las acciones. Darle este triple enfoque al diagnóstico, no significa abordar necesariamente todos los aspectos del fenómeno, sino que se puede determinar uno, o unos pocos, para profundizar en ellos, pero en cada uno, teniendo en cuenta estos tres puntos de enfoque que son los que garantizan una visión de conjunto realmente abarcadora [...]. Es necesario realizar diagnósticos participativos e integrales de los sujetos y los procesos involucrados en el acto pedagógico preferiblemente con triple diagnóstico. (pp. 25-26)

C. Núñez (1998) también se refiere al diagnóstico como "la entrada" para poder partir de la práctica,

entendida no solo como lo que "se hace", sino como lo resultante de la confrontación entre la concepción del mundo, la interpretación del contexto y lo que en realidad se hace, o se deja de hacer, destacando la incoherencia que, en ocasiones se hace patente entre lo que pensamos lo que hacemos y lo que es resultado real de nuestras acciones. A este tipo de diagnóstico se le conoce como "triple diagnóstico" (que en dependencia al nivel de participación que se alcance pudiera llegar a ser un triple autodiagnóstico). (p. 25)

Este diagnóstico, que permite centrarse en el sujeto, reconoce tres elementos:

- El individuo con sus conocimientos, motivaciones, relaciones, concepción del mundo y proyecto de vida.

- El medio social en que se desenvuelven las relaciones: grupo estudiantil, profesores, familia, amigos, etc.

- $\quad$ El modelo que el proceso educativo desea alcanzar en el estudiante.

Además, busca información sobre tres aspectos fundamentales:

- La realidad objetiva en que vive y actúa el estudiante. 
- El accionar o actuar grupal e individual consciente o espontáneo (lo que se hace y se dice).

- La interpretación consciente de la realidad (lo que se piensa).

En consecuencia, la investigación reconoce la multiplicidad de aspectos pertenecientes al mundo social y, en atención a ello, se centra en los comportamientos valorativos que, desde la práctica educativa elegida para el trabajo de campo (servicio de régimen interno), constituyen el escenario que da cuenta de los valores implicados en la educación.

Para la recolección de los datos se utilizaron dos técnicas de orden cualitativo, estas fueron, como se mencionó anteriormente: la observación encubierta y el análisis epistolar. Respecto a la observación encubierta, desde la perspectiva de Corbetta (2007), se trata de una variante de la observación participante. La justificación fundamental para defender su uso es el hecho de que, cuando se sabe observado, el ser humano se comporta, presumiblemente, de forma distinta a la habitual. En consecuencia, el tipo de observación que se plantea permite captar de forma más genuina el modo de actuar natural.

Específicamente dentro del ejercicio investigativo, base de la ruta propuesta, este tipo de técnica permitió el levantamiento de la información en la población de alféreces estudiada en su medio natural. Con esto se buscaba describir el comportamiento del participante en los roles del servicio del régimen interno elegidos. La guía de observación permitió recoger los registros de las actividades de cada uno de los equipos de investigadores (ver Apéndice A).

El servicio de régimen interno consiste en el conjunto de actividades no propias al cargo, que desarrollan los miembros de una unidad militar para garantizar su correcto funcionamiento. Se define como el "conjunto de disposiciones que regulan el funcionamiento de una unidad. Está normalizado por el Reglamento de Servicios de Régimen Interno (Comando General de las Fuerzas Militares, (s. f.), el cual se aplica como ejercicio de la práctica militar formativa de los cadetes y alféreces de la Escuela Militar.

La aplicación de la observación encubierta tuvo las siguientes características:

- Número de investigadores: seis, que constituyeron tres grupos de observación. 
- Los sujetos-objetos de investigación fueron estudiantes alféreces de octavo semestre en fase de mando que, por encontrarse en el último nivel de formación, poseen experiencia en las actividades señaladas y se adiestran en la ejecución del servicio de régimen interno.

- Se seleccionaron seis tipos diferentes de servicios de régimen interno, de los cuales cada grupo observó dos. Estos fueron: alférez de escuela, alférez control aulas, alférez comandante guardia principal, alférez relevante guardia principal, alférez relevante guardia occidental y alférez control enfermería.

- El tiempo de observación por servicio fue de dos horas, lo que significó cuatro horas en cada servicio y dieciséis horas de observación por grupo, lo que equivale a cuatro observaciones por servicio. De este modo se completó un total de 24 estudiantes observados, que representan el $11.11 \%$ de alféreces de una población de 216 que hacen parte del octavo semestre.

En cuanto al uso de la técnica epistolar o carta preescrita dentro del proceso investigativo, Corbetta (2007) señala que esta se emplea para describir las formas tradicionales de vida, para ello se trata de centrar la atención en los aspectos subjetivos de los procesos sociales, en particular, en los mecanismos de adaptación de los individuos en una sociedad en vías de trasformación. En la investigación, se utiliza para conocer las comprensiones de los estudiantes sobre el respeto y la honestidad a través de los indicadores de evaluación, lo que piensan, sienten y sus actitudes, a través de lo que expresan escrituralmente en la carta a completar. El estudio no se refiere a cada estudiante de manera individual, pero sí muestra una tendencia sobre la comprensión y las actitudes presentes en el grupo de estudiantes respecto del desarrollo del respeto y la honestidad profesional. Entre las características de su aplicación están las siguientes:

- La técnica de análisis epistolar se desarrolló mediante el completamiento de una carta preescrita, diseñada desde los indicadores de los valores de respeto y honestidad (ver Apéndice B).

- Se aplicó a veinticinco estudiantes de octavo semestre en fase de mando, escogidos aleatoriamente. 


\section{Referencias}

Astorga, A. (1994). Manual de diagnóstico participativo. En L. Vargas y G. Bustillos, Técnicas participativas para la Educación Popular. Tomo I (2.a ed.), (s. p.). Lumen-Humanitas y Cedepo.

Corbetta P. (2007). Metodología y técnicas de investigación social. McGraw Hill.

Comando General de las Fuerzas Armadas (s. f.). Reglamento de Servicio de Guarnición (6. ${ }^{\text {a }}$ ed.). Comando General de las Fuerzas Armadas.

De la Rúa, M. J. (2013). El diagnóstico pedagógico como proceso pedagógico. https://www. researchgate.net/publication/263653690_El_Diagnostico_Pedagogico_como_proceso_ pedagogico_y_participativo_de_acercamiento_a_la_realidad_educativa

Ejército Nacional de Colombia (2009). Reglamento de Régimen Interno para Unidades Tácticas EJC-3-22-1.

Ejército Nacional de Colombia (2016). Doctrina Militar. Manual Fundamental del Ejército MFE 1-0.

Escuela Militar de Cadetes “General José María Córdova” (ESMIC). (2019). Proyecto Educativo del Programa de Profesional en Ciencias Militares (PEP).

Guervilla, E. (1994). Valores y contravalores. Revista Vela Mayor, 1(2), 32.

Núñez, C. (1998). La Revolución Ética. Indec.

Sánchez, A. (2005). Análisis filosófico del concepto valor. Humanidades Médicas, 5(2), 1-23. http:// scielo.sld.cu/scielo.php?script=sci_abstractypid=S1727-81202005000200009ylng=esynr$\mathrm{m}=\mathrm{isoyt} \operatorname{lng}=\mathrm{es}$

Sandoval, M. (2007). Sociología de los valores y juventud. https://www.researchgate.net/publication/26496561_Sociologia_de_los_valores_y_juventud 